\title{
Sleep and circadian rhythms in mood disorders
}

Armitage R. Sleep and circadian rhythms in mood disorders.

Objective: Self-reported sleep disturbances are present in over $80 \%$ of patients with depression. However, sleep electroencephalography (EEG) findings, based on overnight polysomnography have not always differentiated depressed patients from healthy individuals.

Method: The present paper will review the findings on sleep EEG studies in depression highlighting how recent technological and methodological advances have impacted on study outcomes.

Results: The majority of studies, including our own work, do indicate that sleep homeostasis and sleep EEG rhythms are abnormal in depression, but the sleep disturbances were strongly moderated by gender and age. Melancholic features of depression correlated significantly with low slow-wave activity in depressed men, but not in depressed women. Women with depression showed low temporal coherence of sleep EEG rhythms but the presence or absence of melancholic features did not influence correlations.

Conclusion: Diagnostic classification schemas and clinical features of depression may influence sleep EEG findings, but gender may be a more important consideration.

\section{R. Armitage}

Department of Psychiatry, University of Michigan, Ann Arbor, MI, USA

Prof. Armitage declares no conflict of interests.

Key words: sleep; depression; mood; melancholia; review of the literature

R. Armitage, University of Michigan, Sleep and Chronophysiology Laboratory, 2101 Commonwealth, Suite D, Ann Arbor, MI 48105, USA.

E-mail: rosearmi@med.umich.edu

Presented at the Conference: 'Melancholia: Beyond DSM, Beyond Neurotransmitters', May 2-4, 2006, Copenhagen, Denmark.

\section{Summations}

- Sleep EEG abnormalities are evident in depression but are strongly influenced by age and gender. For the most part, sleep microarchitecture, based on sleep EEG frequency analysis, is more likely to differentiate depressed patients from healthy controls than are standard measures of rapid eye movement latency or insomnia.

- Men with depression show reduced slow-wave activity in non-rapid eye movement sleep and impaired homeostatic response to sleep challenge. Women with depression show low temporal coherence in sleep EEG rhythms. Additional data indicate that low temporal coherence may be a risk factor for depression in females evident before the onset of the first episode.

- Diagnostic classifications and clinical features of depression are also important considerations in sleep studies; however, melancholic features of depression were strongly correlated with sleep abnormalities only in men but not in women.

\section{Considerations}

- Some of the studies discussed in the paper included only small sample sizes with the inclusion of broad age groups. To fully assess gender and age-related changes in sleep, large-scale studies are necessary.

- Few studies include longitudinal assessment of sleep EEG. These data are essential in determining the clinical utility of sleep EEG in predicting course of illness.

- Sleep, melancholia, and the issue of age of onset and period of greatest risk for depression needs to be revisited. 


\section{Introduction}

Interest in the relationship between sleep abnormalities and depression has a rich history with more than 1500 published articles since the 1960s. This paper will review the studies of sleep abnormalities in patients with depression and discuss how moderating variables such as age, gender, diagnostic typologies and patient subtypes have influenced results. The ways in which technological and theoretical advances in sleep research have contributed to understanding the pathophysiology of depression will also be discussed.

\section{Nomenclature}

As the nomenclature for describing sleep physiology differs across studies, we define several key terms that will be used throughout the remainder of this paper. Sleep macroarchitecture refers to the measures derived from visual stage-scoring of sleep electroencephalography (EEG) including sleep latency, rapid eye movement (REM) latency, total sleep time, and the minutes and percentages of stages 1-4 of non-rapid eye movement (NREM) sleep, REM and awake. Sleep microarchitecture denotes the parameters derived from all-night, computerized, quantitative EEG analyses including incidence, amplitude and power in five standard EEG bands: beta, sigma, alpha, theta and delta.

Slow-wave sleep refers to stages 3 and 4 of NREM sleep and specifically requires the presence of very high amplitude delta waves $(>75 \mu \mathrm{V})$ for more than $20 \%$ of each $30 \mathrm{~s}$ epoch for stage 3 and more than $50 \%$ of the epoch to define stage 4 sleep, according to standard criteria (1). Slow-wave activity (SWA) refers to delta, amplitude and power in NREM sleep and requires neither a minimum amplitude nor a percentage of the epoch criterion. Slow-wave sleep is concentrated in the first half of the night and is not usually evident in the latter half in adults (2). Note, however, that SWA does recur even in late NREM sleep periods. Throughout the night, delta amplitude and power show a very consistent 90 min ultradian rhythm, although amplitude and power are highest in the first or second NREM period and decline over the night. Thus, the dynamics and temporal organization of SWA differ substantially from slow-wave sleep measures (3-8).

Theoretical views of sleep abnormalities in depression

The majority of studies on macroarchitecture have reported abnormalities in the timing and/or distribution of REM and NREM sleep stages as primary characteristics of those with major depressive disorders (MDD). Specifically, early REM onset (i.e. short REM latency, REML) increased REM time and decreased slow-wave sleep have been described in MDD. Most of the current theories regarding the mechanisms underlying sleep disturbances in MDD assume that there is a reciprocal relationship between REM and slowwave sleep control mechanisms, such that a shorter latency to the first REM period results in reduced slow-wave time in the first NREM period. The issue that remains debated is whether the primary disturbance is one of REM or slow-wave regulation. McCarley (9) postulated that sleep disturbances in MDD were largely because of increased cholinergic activation that resulted in the early onset of REM sleep. This model, based on animals studies $(10,11)$, assumes that cholinergic neurons in the pontine reticular formation of the brain stem are activated during REM sleep, whereas noradrenergic/serotonergic neurons in the locus coeruleus and dorsal raphe are deactivated. Thus, the neurotransmitter systems involved in REM sleep control are reciprocal and responsible in part, for subsequent REM/NREM sleep cycle oscillations. He reasoned that an early onset of REM sleep in MDD (i.e. short REML) reflects an imbalance in normal cholinergic/aminergic neurotransmission that produces disinhibition of REM sleep (9). There has been fairly strong support that the administration of cholinergic agonists indeed triggers an earlier onset of REM in both animal and human studies (cf. 12-16). However, establishing an even greater cholinergic response during sleep in those with MDD has been more equivocal (17-19). Most studies have shown a shorter mean REML in response to cholinergic challenge in those with MDD, compared with healthy controls. However, the reported inter-individual variability within both MDD and control groups is often extremely large $(13,14,16)$. Nevertheless, there is little evidence to refute increased cholinergic activation as a model of sleep disturbance in MDD.

An alternative approach to understanding sleep disturbances in MDD has focused on NREM sleep. Rather than REM disinhibition, Borbély and colleagues have suggested that sleep regulation and homeostasis is impaired in those with MDD (20, 21). This hypothesis is based on the two-process model of sleep regulation $(22,23)$, where the amount of SWA at night is determined by the amount of prior wakefulness, the level of sleep propensity during the day and depth of sleep at night (Process S). Process S accumulates during waking hours as slow-wave sleep propensity rises 
and dissipates over the night. Measuring the temporal evolution of SWA during NREM sleep is presumed to approximate Process $\mathrm{S}$ and thus, there is an exponential decline in SWA across successive NREM sleep periods. The second process (Process C) reflects the circadian control of sleep propensity that is highest at 3-5 a.m. when it is very difficult to overcome the need to sleep and is lowest at 4 p.m., where sleep propensity is minimal. Process $\mathrm{C}$ is presumed to reflect internal clock control of circadian rhythms, the threshold for maintaining wakefulness and REM sleep control, and is unaffected by the amount of prior wakefulness. It is assumed that in a healthy brain, the propensity for REM sleep increases as SWA dissipates, thus explaining the short duration first REM period when SWA pressure is high, and the increase in REM as SWA dissipates across the night $(22,23)$. Thus, the time course of SWA approximates Process $\mathrm{S}$, not the total amount of SWA.

With regard to MDD, Borbély \& Wirz-Justice (23) proposed that the reduction in slow-wave sleep reported in some patients with MDD resulted from impairment in Process S, with diminished accumulation of sleep pressure during the daytime and reduced dissipation of SWA at night. Thus, both slow-wave sleep time and SWA are reduced in the initial NREM sleep period, allowing an earlier onset of the first REM period with a longer duration. In a later review article Wirz-Justice (24) further postulated that extending prior wakefulness or sleep deprivation would normalize SWA regulation and Process S in MDD. Note, however, that the only evidence cited in support of the proposed Process S deficiency in MDD was the antidepressant effects of sleep deprivation and relapse after recovery sleep and reduced slowwave sleep time coupled with short REM (21). As pointed out by Wirz-Justice (24) and van den Hoofdakker and colleague $(25,26)$ the hypothesized impairment in the regulation of SWA has not been adequately tested.

Both the Process S deficiency hypothesis and the increased cholinergic activation model of MDD share the same primary assumption, that the timing and duration of REM and slow-wave sleep are reciprocal and interactive, and that increasing one will result in a compensatory decrease in the other. Moreover, this same reciprocal relationship is assumed to underlie REM and slow-wave sleep cycles in healthy adults. Interestingly, this assumption is not usually tested empirically, with two notable exceptions. Although not a direct test of the Process S deficiency hypothesis, van den Hoofdakker and colleague have tested the assumption that REM and slow-wave sleep are reciprocal. In the first study, the production rate of slow-wave sleep was evaluated in nights with sleep onset REM periods (SOREM) vs. nights with 'normal' REML. They reasoned that the accumulation of slow-wave sleep time in the SOREM nights would be lower than on normal nights if REM and slow-wave sleep were reciprocal. No differences in slow-wave sleep accumulation were found between short and normal REML nights (25). Further, Schulz and Lund (27) argued that if short REML was the result of decreased slow-wave sleep propensity, then the amount of slow-wave time should correlate with REML in patients with MDD. Significant correlations were not found. Results from our own work also indicate no correlation between REML and SWA in the first sleep cycle in either patients with MDD or healthy adults (28). These data strongly suggest that REM and SWA in the first sleep cycle are not reciprocal but do not address whether SWA is reduced or if the regulation of SWA is impaired in those with MDD. Only a handful of studies have evaluated SWA in MDD and to our knowledge, only one study has examined the regulation of SWA in MDD (29).

Nevertheless, the Process S deficiency model has several benefits over the increased cholinergic sensitivity hypothesis. First, the former approach accounts for abnormalities in the timing of sleep onset, intermittent awakenings during the night, decreased SWA as well as decreased slow-wave sleep and REM timing abnormalities. Secondly, it allows specific predictions about the effects of manipulating prior wakefulness, sleep restriction and deprivation, and thus permits an evaluation of sleep regulatory control mechanisms rather than simply providing a descriptive explanation for changes in sleep architecture. The cholinergic hypothesis accounts for REM sleep timing abnormalities but does not address NREM sleep abnormalities per se except as a consequence of REM disinhibition. Further, increased light stage 1 sleep and intermittent wakefulness are not addressed by McCarley (9). More recent revisions of reciprocal interaction models of normal sleep do allow for intrusions of wakefulness but the applicability to sleep in MDD has yet to be established $(30,31)$. There are also practical limitations to testing the cholinergic sensitivity hypothesis, namely that cholinomimetics are often associated with significant and fairly long-lasting side-effects including nausea and vomiting and the discomfort and sleep disturbing effects of sleeping with an indwelling catheter. Further, the applicability of the cholinergic model in MDD depends on the presence of a 
REM sleep abnormality. Impairment in the regulation of SWA does not depend on the presence of short REML nor is the amount of SWA in the first sleep cycle correlated with REML $(28,32)$. Most importantly, assessing whether sleep regulation and homeostasis is impaired in depression requires a sleep challenge paradigm where SWA response is assessed.

Nevertheless, both models of sleep regulation continue to be viable explanations of sleep EEG abnormalities in depression research. We will now review the findings on sleep abnormalities in depression.

\section{Subjective sleep disturbances}

Sleep disturbances are key features of depressive symptomatology, with subjective sleep complaints in more than $80 \%$ of depressed patients (33). Historically, insomnia has been the most pervasive self-reported sleep disturbance appearing in the literature as hyposomnia as early as the 1920s (cf. 34) and continuing with classifications of sleep onset insomnia and early morning awakenings with an inability to return to sleep in later studies (33). There are numerous studies that highlight the prevalence of insomnia in those with mood disorders (35) and the prevalence of depression in those with insomnia complaints (36-38). Further, those with both sleep onset and increased nocturnal awakenings (i.e. sleep maintenance insomnia) had greater symptoms of depression than those with either sleep onset or sleep maintenance insomnia alone (39). Persistent insomnia has also been linked to increased risk of relapse and recurrence of depression and to increased risk of suicide in adults $(35,40,41)$ and in adolescents with depression (42, 43).

There is also ample recognition of the relationship between sleep and depression in almost all diagnostic and symptom severity assessments in use worldwide (44). It should be noted however that most of the depression severity rating scales focus more on insomnia or reduced total sleep time including both the Montgomery-Asberg, and Hamilton Depression Rating Scales. However, insomnia is not the only sleep complaint in those with depression. It is estimated that $15-35 \%$ of patients complain of hypersomnia, and difficulty getting up in the morning, although most studies suggest that this is more characteristic of atypical features or in patients with bipolar illness (45-48). A recent study by Parker et al. (49) indicated that hypersomnia was more prevalent than early morning awakening in bipolar and both melancholic and non-melancholic unipolar depressed patients.
Prevalence of hypersomnia decreased with age in all subtypes of depression, but it was only the melancholic and bipolar patients who showed an increase in early morning awakening with increasing age of patients (49).

Taken together, these findings highlight the prevalence of self-reported sleep disturbance and the association between persistent sleep problems and a poor clinical course, regardless of whether the primary complaint is hypersomnia or insomnia. For the most part, objective sleep measures based on laboratory sleep EEG and polysomnography (PSG) have been considered among the most reliable biological features of depression.

\section{Laboratory-based sleep studies}

Sleep macroarchitecture

Numerous overviews of normal sleep macroarchitecture, based on visual scoring of sleep EEG, have been published in the last 30 years (50). In healthy adults, sleep onset occurs 10-15 min after lights out, followed shortly thereafter by the progression of deeper stages 2, 3 and 4 of NREM sleep. The first REM period terminates the first slow-wave sleep (stages 3 and 4) episode and occurs about 90 min after sleep onset. The first REM period is short in duration (1-5 min) but lengthens progressively across successive REM periods. NREM and REM sleep alternate throughout the night at about an 80-120 min period length. However, slow-wave sleep is concentrated in the beginning of the night and is not present in abundance in the latter half except in younger adolescents and children. Short duration intermittent awakenings do occur in both healthy children and adults, but generally only 2 $5 \%$ of total sleep time.

The 'classic' laboratory sleep profile of a symptomatic, but unmedicated, adult with MDD is a prolonged sleep latency (sleep onset insomnia), bouts of intermittent wakefulness (middle insomnia), increased light, non-restorative stage 1 sleep, decreased slow-wave sleep, a shortened latency to the first REM period accompanied by increased phasic REM density and an early morning awakening, also known as late or terminal insomnia (3, 50-52). However, early research assumed that insomnia and sleep fragmentation were non-specific characteristics of sleep in a variety of psychiatric illnesses, but that REM sleep abnormalities were more specific to depression (53-55). Several studies indicated that significantly shorter REML, a prolonged first REM sleep period and increased phasic REM activity were characteristic of those with primary depression $(56,57)$. In fact, much of the 


\section{Armitage}

early work from the Pittsburgh group pointed to short REML (REML) as a biological marker for depression (58), prompting a long-standing interest in sleep abnormalities in psychiatric illness and a tremendous growth in laboratory studies of depression (59).

Subsequent work has indicated that using a cutoff of 65 min may provide the most sensitive and specific discriminator those with short REML (60), and one that best correlates with terminal insomnia appetite loss and unreactive mood (61). In addition, Rush and colleagues also showed that short REML predicted antidepressant treatment response to tricyclics $(62,63)$ and to placebo nonresponse (64). Short REML may also persist into clinical remission (65-67) and may also be evident in the first-degree non-affected relatives of depressed patients $(68,69)$. Roughly half of those who show short REML also show dexamethasone non-response (70), particularly among endogenous depressed patients (65). In addition, short REML may be more prevalent in acute, recurrent depression $(71,72)$ and are associated with a worse clinical course with greater risk of relapse and recurrence (73).

However, REM sleep findings have been much less consistent in recent studies. A meta- analysis of 177 published studies indicated that short REML was not specific to depression and that no single sleep measure reliably differentiated patients from controls (74). Lauer et al. (75) has concluded that REM sleep abnormalities in depression are not evident until the fourth decade of life. Our own studies have failed to demonstrate significantly shorter REML in depressed adults regardless of age $(28,32,76,77)$ and for the most part in depressed children and adolescents (78). The issue of age-related changes in sleep EEG is discussed in greater length below. On the other hand, computer analysis of sleep EEG frequencies, or sleep microarchitecture, has been more successful in differentiating patients form controls $(4,79)$.

\section{Sleep microarchitecture}

There is some evidence that increased fast-frequency beta activity and elevated alpha are present during sleep in depressed patients $(52,76,80-82)$, consistent with hyperarousal and increased sleep fragmentation among depressed patients. These findings are also in agreement with PET brain imaging studies demonstrating a lower decrease in relative regional cerebral glucose metabolism from the pre-sleep period to NREM sleep (83). However, there are a few studies that suggest that beta activity may be higher in primary insomnia than in depression (84). On the other hand, the finding of increased fast frequency EEG activity in depression is also consistent with waking EEG studies (85).

Nonetheless, there is additional evidence that the synchronization of EEG activity during sleep is compromised in depression. Temporal coherence of sleep EEG activity recorded from the right and left hemispheres and in the synchronization of fast and slow frequency EEG is significantly lower in depressed adults (76) and adolescents $(78,86)$. Similar results have been obtained in the assessment of waking EEG activity and coherence among electrode site (87).

In addition, lower temporal coherence and decreased synchronization of fast frequency activity during sleep may also be evident whether patients are symptomatic and unmedicated or in clinical remission (82) suggesting that these sleep microarchitectural abnormalities may be trait-like features of the illness. Moreover, low temporal coherence is also present in those at high-risk for depression based on family history (88) but particularly among adolescent girls with a maternal family history of depression (89). The latter study also showed a greater risk for onset of depressive symptoms 3-5 years later in those high-risk girls with the lowest temporal coherence. These findings are in general agreement with those of Modell et al. $(90)$ and Giles et al. $(68,69)$ focusing on short REML as a risk factor for subsequent depression. Unfortunately, we have not shown a significant relationship between REML and temporal coherence in any of our studies but continue to show consistently lower temporal coherence among females with depression, regardless of age.

Other studies have shown that it is primarily SWA in NREM sleep that differentiates patients from controls. Borbély et al. (21) demonstrated lower SWA in nine adult unipolar, unmedicated, depressed patients compared with age- and gendermatched healthy normal controls. This initial finding was confirmed in a group of younger patients with MDD (20-30 years old), compared with age-matched controls (91). SWA did not, however, differentiate older patients ( $>50$ years) from gender-matched controls in the same age range (92), and may not be a characteristic of bipolar patients (93).

Kupfer et al. (94) have also utilized delta wavecount statistics, based on period amplitude analysis to quantify the incidence of SWA in NREM sleep. In several studies, they have reported lower delta wave counts in patients with MDD compared with controls, and these differences were largely restricted to the first NREM period of the night (94). In fact, Kupfer's group has suggested that 
delta wave counts in patients with MDD are higher in the second NREM period than in the first. It has also been suggested that this delta ratio is related to the clinical course of illness and symptom severity (50, 94-96). Other studies have failed to confirm higher SWA in the second NREM period in depressed patients (77). Most subsequent studies have demonstrated lower SWA in NREM sleep in depression $(28,32)$, with one exception (97).

Considering both historic and more recent work, it appears that earlier studies were much more likely to show significantly shorter REML, sleep onset insomnia and early morning awakenings. Although findings have been more robust with sleep microarchitecture measures, they are not entirely consistent across studies. Regardless of whether it is SWA in NREM sleep, a distinct EEG profile during REM sleep or low temporal coherence that identifies patients with depression, all these findings do suggest that abnormalities in the timing of the REM/NREM sleep cycle and more globally in the organization of the sleep/wake cycle are core features of depression. There are, however, a number of methodological issues and procedural changes that are likely to contribute to discrepancies among studies.

\section{Factors influencing sleep abnormalities in depression}

Age and sex effects

Interestingly, the studies which have failed to show SWA differences between patients and controls have largely included middle-aged depressed patients $(97,98)$. Although the early work of the Pittsburgh group (Reynolds et al., 1983) had suggested that sleep disturbances were more profound in older depressed patients, more recent data have shown that differences between patients and controls may be more prevalent among younger adults and diminish with increasing age $(28,32)$. As circadian rhythms tend to phase advance, REML gets shorter and more general sleep disturbance increases with age in healthy individuals, it is no surprise that studies are less likely to differentiate between patients and controls. Clearly, however, this is not the case with very early onset depression as few studies show consistent sleep EEG abnormalities in children with depression (cf. 100). Both sleep macro- and micro-architectural abnormalities are more prevalent in adolescents with depression but are also strongly moderated by gender $(78,86)$. In addition to lower temporal coherence, females with depression are also more likely to show significantly weaker circadian amplitude than depressed males, and spend significantly less time in bright light (101). Importantly, low circadian amplitude was also evident even in 8- to 12-yearold depressed girls, one of the few studies to identify abnormalities in young depressed patients. On the other hand, boys with depression were more likely to show reduced slow-wave sleep and short REML than girls, but only among adolescents. Other studies in adults also indicate that age and gender are important moderating variables.

In a large-scale study of 302 MDD men and women, Reynolds et al. (102) demonstrated significant gender main effects for slow-wave sleep and delta wave counts across the whole night and particularly in the first NREM sleep period. Men with MDD had less slow-wave sleep and lower delta counts than women with MDD. Moreover, a NREM period by gender interaction was found, suggesting that the temporal distribution of delta wave counts differed for men and women with MDD. Gender differences in both slow-wave sleep and delta wave were also evident in younger patients (20-29 years old) with MDD and diminished with increasing age, though not monotonically. Reynolds et al. concluded that age effects were stronger than gender differences and there was little evidence of a differential maturational time course in men and women with MDD. Published reports from our own group are not consistent with the interpretation of Reynolds et al. The discrepancy, however, can be easily reconciled. First, in the study of Reynolds et al., delta wave counts were lower in both younger and older MDD men than in women. Thus, women with MDD retained more delta even later in life, providing evidence that the maturational time course differs from men with depression and that gender differences in the pathophysiology of depression may persist throughout adulthood.

Several of the recent studies in our laboratory have focused on gender differences in SWA regulation in patients and controls. The initial study of 22 patients and 23 controls revealed significant group by gender interactions on the amplitude and power of SWA across successive NREM periods, and SWA time course (103). Men with MDD had a significantly slower rate of decay with lower predicted SWA power and amplitude parameters from exponential regression analysis, compared with all other groups. Women with MDD, healthy men and healthy women did not differ from each other. Most importantly, a comparison study with schizophrenic men indicate that abnormalities in the time course of SWA were specific to MDD. Although some men with schizophrenia showed lower SWA amplitude than healthy men, they did not differ on the accumulation or dissipation of 
SWA over NREM sleep time. The men with MDD, on the other hand, differed significantly $(P<0.05)$ from both healthy and schizophrenic men.

That baseline SWA was more likely to be abnormal in men with MDD was replicated in a follow-up study of 131 subjects, 20-40 years of age (104). Analysis of SWA across successive NREM periods produced a significant group by gender interaction, although this effect was largely restricted to the first NREM period. Again, SWA in the men with MDD was significantly lower compared with all other groups, with no significant differences among NC men and women and women with MDD. Approximately $70 \%$ of men with MDD fell below mean SWA in the control group. By contrast, only $20 \%$ of women with MDD fell below the mean SWA for healthy women. Exponential regression analyses confirmed that the time course of SWA was abnormal in men with MDD whereas no differences were found among $\mathrm{NC}$ men and women, and women with MDD. Men with MDD showed both lower predicted SWA with a slower accumulation and dissipation across the night. Regression parameter estimates in men with MDD were outside the $95 \%$ confidence intervals of all other groups $(P<0.05)$, whereas normal control men and women and MDD women did not differ from each other. It is difficult to reconcile these findings with the suggestion that gender differences are because of factors such as skull thickness as both disease- and sex-dependent components were identified. If gender differences were due simply to divergent electrical conductivity, then no interaction with group should be evident. Further, both the time course and amplitude differentiated men with MDD from all other groups. Such findings make a stronger case for sleep regulatory processes that are influenced by both disease and gender. The definitive test of the SWA regulation hypothesis would require directly manipulating prior wakefulness and homeostatic regulation of SWA. Although numerous sleep deprivation studies have been conducted in patients with MDD, only one study outside our own work has evaluated delta response to sleep deprivation (97). This small study $(n=7)$ indicated little change in delta activity after sleep deprivation, supporting impaired sleep homeostasis.

An ongoing study of SWA homeostasis in our own group has shown a blunted SWA response to a 3-h sleep delay in men with depression in addition to reduced total SWA power on both baseline and delay nights compared with healthy men and women. By contrast, women with depression showed the largest response to sleep challenge,

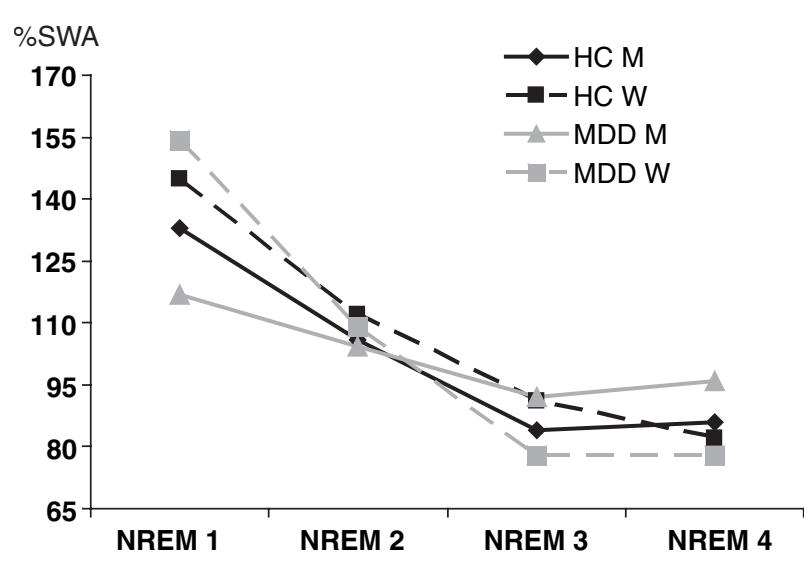

Fig. 1. Slow-wave activity (SWA) response to a 3-h sleep delay in the first four non-rapid eye movement (NREM) sleep periods in healthy control men (HC M), healthy control women $(\mathrm{HC} \mathrm{W})$, depressed men (MDD M) and depressed women (MDD W), expressed relative to baseline SWA.

supported by a significant group by sex interaction, as seen in Fig. 1. These results indicate that SWA regulation and sleep homeostasis are impaired in depression, but add the caveat that is heavily sexdependent. As this study included only 20 - to $40-$ year olds, it is not clear whether those younger and older would show the same sex-dependent homeostatic abnormalities and whether a complete sleep deprivation protocol would elicit a larger SWA response in the depressed men (105).

Taken together, these findings strongly support a differing pathophysiology of depression in males and females. Males with depression are more likely to show impairment in sleep homeostasis, whereas females are more likely to show biological rhythm abnormalities and chaotic sleep EEG organization. The maturational changes in sleep EEG may also be sex-specific.

In summary, a number of methodological and theoretical developments in sleep research have permitted a more refined assessment of sleep abnormalities in depression. It certainly appears that some microarchitectural features of depression are present during an index episode, in clinical remission and are evident in those at high risk for depression. However, there are also clinical issues that influence study outcomes.

Diagnostic features and subtypes of depression

One the one hand, the Diagnostic and Statistical Manual of Mental Disorders (DSM) has lead to more uniform and standardized procedures for the diagnosis of depression, but it may have contributed to a decreased likelihood of detecting significant sleep abnormalities in depression. For 
example, earlier studies that included only melancholic patients or endogenous depressed patients were more likely to differentiate patients from controls than subsequent work focusing on major depressive illness from DSM-III and DSM-IV. It is not clear however, to what degree diagnostic schema and methodological differences have contributed to the discrepancies between the early and more recent work.

As discussed above, there is also good evidence that insomnia in the extreme is associated with mania and the hypersomnia often accompanies the depressed phase in bipolar patients. Moreover, one would expect more severe sleep disturbance, particularly insomnia, in melancholic patients in whom early morning awakening is a key feature (49). One would also expect increased REM sleep among those with loss of appetite and weight loss (61), and perhaps greater fast-frequency EEG activity, indicative of increased arousal (4). Loss of homeostatic sleep drive might also be more prevalent in the more severe, anhedonic, psychomotor-retarded patients. Part of the difficulty in determining the source of variance between older and newer sleep studies is that few recent sleep studies assess melancholic features or include psychotic depression.

To try and address part of this issue, we used our data archive of sleep EEG studies to conduct a preliminary study of which features of depression correlated with SWA and biological rhythm abnormalities. Low SWA and an abnormal time course was associated with increased suicidality (ideation and attempts) and higher symptom severity. Moreover, those with the lowest SWA also showed the greatest psychomotor retardation. REM sleep measures were not strongly correlated with clinical features of depression. These findings are consistent with impaired sleep homeostasis in more severe depression. However, the effect was significantly stronger in males than in females ( $r=-0.38$ vs. $r=-0.29$, respectively). On the other hand, low temporal coherence was associated with an earlier age of onset of depression, positive family history of depression and incomplete inter-episode recovery. The sex differences in SWA and temporal coherence decreased with age as did the correlations between sleep and clinical features and fewer sex differences in sleep or clinical features of depression were evident in those patients over 40 years of age. Further, there was only a slight tendency for sex differences in temporal coherence to be less dramatic in those with melancholic features, but the effect was very small and not statistically significant.
Most importantly, SWA regulation was significantly related to melancholia in men but neither temporal coherence nor SWA not in women was influenced by melancholic features. Melancholic men had the lowest accumulation of SWA and flatter dissipation across NREM sleep than either melancholic women or non-melancholic subjects of either gender $(P<0.004)$. Women with melancholic features showed a higher accumulation of SWA than non-melancholic women, but it was not outside the $95 \%$ confidence interval. There was also no significant difference in temporal coherence between those women with and without melancholic features $(F<1)$. In short, not only is impaired sleep homeostasis more likely in depressed men, but it is most impaired in those men with melancholic features. This does not appear to be the case in depressed women. Taken together, these data strongly suggest that it is not melancholic symptoms per se that are associated with the sleep disturbance, but is the interaction with gender. However, all of the patients included in the studies from our archival analysis and from all of our published studies, were classified according to DSM-III and DSM-IV criteria and thus the discussion of melancholia, sleep and gender above is restricted entirely to melancholia as a specifier for major depressive disorder. Unfortunately, there are two few recent studies to determine if our findings generalize to the broader conceptualization of melancholia. However, there is growing support that clinical features of depression differ between men and women, well beyond biological measures.

In a recent sample of over 1400 depressed patients, recurrent depression was more prevalent in women than in men, and women had more episodes of depression over their lifetime (104). Positive family history was also more prevalent in women with depression accompanied by an early age of onset of the first episode of depression (105). These findings are consistent with our own archival analysis pointing to more disturbed sleep EEG rhythms in women with a positive family history of depression and a worse clinical course. This, coupled with the findings of the Morehouse et al. (89) study, strongly suggest that low temporal coherence is a strong biological risk factor for depression that is sex specific. It is possible that low SWA and impaired sleep homeostasis is a primary biological risk factor for depression in males, although this awaits further exploration. There is heuristic appeal to view depression as the mood response to the loss of appetitive drive and homeostasis.

It is also not clear whether bipolar illness is associated with the similar sleep abnormalities. The 
majority of studies have shown that sleep loss or sleep restriction is associated with an increase in manic symptoms and that extreme insomnia characterizes a manic episode (108). Our own data has shown that bipolar patients in the depressed phase are more likely to extended sleep and hypersomnia, than are unipolar patients (cf. 109). Very early onset bipolar illness in childhood has been associated with significant insomnia, a reduction in sleep continuity and more awakenings than control children (110). These data are quite distinct from the findings in unipolar depression discussed above, where sleep abnormalities are not evident until adolescence (78). Further, some studies suggest that the degree of insomnia predicts risk of future episodes of mania (111). However, other studies have suggested that the degree of sleep disturbance, and insomnia in particular, predicts future episodes of depression in bipolar patients, but does not predict mania (112), and more often in women than in men (113). It is not yet clear whether sleep microarchitectural abnormalities are also evident in bipolar illness. Regardless, subjective sleep assessment and sleep laboratory abnormalities play a key role in identifying the symptoms and risk for depression. Although the diagnostic features and classification of depression does influence sleep EEG abnormalities, gender and age plays as important a role in our view. The distinction between depressive subtypes may become clear as additional evidence on age- and gender-related influences on sleep regulation and biological rhythms and potential sex differences are evaluated across diagnostic classifications.

\section{Acknowledgements}

There are many colleagues who contributed to the studies from our studies cited in this paper. Robert Hoffmann, PhD has been a collaborator for nearly 30 years. He has contributed enormously to the conceptual and empirical work in our lab. I am also grateful to my former colleagues at UTSW in Dallas, TX, John Rush, MD, Madhukar Trivedi, MD, and Graham Emslie, MD and their respective research teams. Our most recent work could not have been completed without the current and ongoing collaborative efforts of Elizabeth Young, MD, Saulo Ribeiro, MD, and Todd Arnedt, PhD. Support for our cited work has been provided by NARSAD, the Stanley Foundation, the David Nathan Meyerson Memorial Fund, and NIMH MH56953, MH61515.

\section{References}

1. Rechtschaffen A, Kales A. (eds). A manual of standardized terminology, techniques and scoring system for sleep stages of human subjects. National Institutes of Health Publication, no. 204. Washington, DC: Public Health Service, U.S. Government Printing Office, 1968.

2. Armitage R. Sleep: biological rhythms and human performance. In: RAMACHANDRAN VS, ed. Encyclopedia of human behavior. San Diego, CA: Academic Press, 1994:205-211.

3. Armitage R, Roffwarg HP. Distribution of period analyzed delta activity during sleep. Sleep 1992;15:556561.

4. Armitage R, Hoffmann R. Sleep electrophysiology of major depressive disorders. Curr Rev Mood Anxiety Disord 1997;1:139-151.

5. Dijk DJ, Beersma DG, Hoofdaker RH. Sex differences in sleep EEG in young adults: visual scoring and spectral analysis. Sleep 1989;12:500-507.

6. Feinberg I, Hibi S, Carlson VR. Changes in EEG amplitude during sleep with age. In: Nancy K, Sherwin J, eds. The aging brain and senile dementia. New York: Plenum Press, 1977:85-98.

7. Feinberg I, March Jd, Fein G, Floyd TC, Walker JM, Price L. Period and amplitude analysis of $0.5-3 \mathrm{c} / \mathrm{sec}$ activity in NREM sleep of young adults. Electroencephalogr Clin Neurophysiol 1978;44:202-213.

8. Feinberg I, Fein G, Floyd TC. Systematic trends across the night in human sleep cycles. Psychophysiology 1979;16:283-291.

9. MCCARLEY RW. REM sleep and depression: common neurobiological control mechanisms. Am J Psychiatry 1982;139:565-570.

10. Hobson JA, McCarley RW, Wyzinski PW. Sleep cycle oscillation: reciprocal discharge by two brain stem neuronal groups. Science 1975;189:55.

11. McCarley RW, Hobson JA. Neuronal excitability modulation over the sleep cycle: a structural and mathematical model. Science 1975; 189:58.

12. Riemann D, Berger M. Sleep, age, depression and the cholinergic REM induction test with RS 86. Prog NeuroPsychopharmacol Biol Psychiatry 1992;16:311-316.

13. Riemann D, Hohagen F, Bahro M, Berger M. Sleep in depression: the influence of age, gender and diagnostic subtype on baseline sleep and the cholinergic REM induction test with RS 86. Eur Arch Psychiatry Clin Neurosci 1994;243:279-290.

14. Riemann D, Hohagen F, Bahro M et al. Cholinergic neurotransmission, REM sleep and depression. J Psychosom Res 1994;38(suppl. 1):15-25.

15. Riemann D, Hohagen F, Fritsch-Montero R et al. Cholinergic and noradrenergic neurotransmission: impact on REM sleep regulation in healthy subjects and depressed patients. Acta Psychiatr Belg 1992;92:151-171.

16. Riemann D, Hohagen F, Krieger $S$ et al. Cholinergic REM induction test: muscarinic supersensitivity underlies polysomnographic findings in both depression and schizophrenia. J Psychiatr Res 1994;28:195-210.

17. Berger M, Riemann D. REM sleep in depression - an overview. J Sleep Res 1993;2:211-223.

18. Berger M, Riemann D, Höchli D, Spiegel R. The cholinergic rapid eye movement sleep induction test with RS-86: state or trait marker for depression? Arch Gen Psychiatry 1989;46:421-428.

19. Gillin JC, Duncan WC, Murphy DL et al. Age-related changes in sleep in depressed and normal subjects. Psychiatry Res 1981;4:73-78.

20. Borbély A. Sleep regulation: circadian rhythm and homeostasis. In: GANTEN D, Pfaff D, eds. Sleep. Clinical and experimental aspects. Berlin: Springer, 1982:83-103.

21. Borbély AA, Baumann F, Brandeis D, Strauch I, Lehmann D. Sleep deprivation: effect on sleep stages and EEG power density in man. Electroencephalogr Clin Neurophysiol 1981;51:483-493. 
22. Borbély A. A two-process model of sleep regulation. Hum Neurobiol 1982;1:195-204.

23. Borbély AA, Wirz-Justice A. Sleep, sleep deprivation and depression. A hypothesis derived from a model of sleep regulation. Hum Neurobiol 1982;1:205-210.

24. Wirz-Justice A. Biological rhythms in mood disorders. In: Bloom FE, Kupfer DJ, eds. Psychopharmacology: the fourth generation of progress. New York: Raven Press, 1995:999-1017.

25. van DEN HoOfdaKKer RH, BeERSMa DG. On the contribution of sleep wake physiology to the explanation and treatment of depression. Psychiatry Res 1986;16:155-163.

26. van den HoOFdakker RH. Chronobiological theories of nonseasonal affective disorders and their implications for treatment. J Biol Rhythms 1994;9:157-183.

27. Schulz H, Lund R. On the origin of early REM episodes in the sleep of depressed patients: a comparison of three hypotheses. Psychiatry Res 1985;16:65-77.

28. Armitage R, Hoffmann R, Fitch T, Trivedi M, Rush AJ. Temporal characteristics of delta activity during NREM sleep in depressed outpatients and healthy adults: group and sex effects. Sleep 2000;23:607-617.

29. BeERSMa DGM, van DEN HoOfdaKKer RH. Spectral analysis of the sleep EEG of depressed patients before and after total sleep deprivation. Sleep Res 1988;17:272.

30. Massaquor SC, McCarley RW. The limit cycle reciprocal interaction model of REM cycle control: new neurobiological structure. J Sleep Res 1992;1:138-143.

31. MCCarley RW, Massaquor SC. Neurobiological structure of the revised limit cycle reciprocal interaction model of REM cycle control. J Sleep Res 1992;1:132-137.

32. Armitage R, Hoffmann R, Trivedi M, Rush AJ. Slow-wave activity in NREM sleep: sex and age effects in depressed outpatients and healthy controls. Psychiatry Res 2000;95:201-213.

33. Reynolds CF, III, KuPfer DJ. Sleep research in affective illness: state of the art circa 1987. Sleep 1987;10:199-215.

34. Kleitman N. Sleep and wakefulness. Chicago, IL: University of Chicago Press, 1963.

35. Ford DE, Kamerow DB. Epidemiologic study of sleep disturbances and psychiatric disorders. An opportunity for prevention? J Am Med Assoc 1989;262:1479-1484.

36. Buysse DJ, Kupfer DJ, Frank E, Monk TH, Ritenour A. Do electroencephalographic sleep studies predict recurrence in depressed patients successfully treated with psychotherapy? Depression 1994;2:105-108.

37. McCall WV, Reboussin BA, Cohen W. Subjective measurement of insomnia and quality of life in depressed inpatients. J Sleep Res 2000;9:43-48.

38. Tan T-L, Kales JD, Kales A, Soldatos CR, Bixler EO. Biopsychobehavioral correlates of insomnia, IV: diagnosis based on DSM-III. Am J Psychiatry 1984;141:357-362.

39. Taylor DJ, Lichstein KL, Durrence HH, Reidel BW, Bush AJ. Epidemiology of insomnia, depression and anxiety. Sleep 2005;28:1362-1363.

40. Breslau N, Roth T, Rosenthal L, Andreski P. Sleep disturbance and psychiatric disorders: a longitudinal epidemiological study of young adults. Biol Psychiatry 1996;39:411-418.

41. Wingard DL, Berkman LF. Mortality risk associated with sleeping patterns among adults. Sleep 1983;6:102-107.

42. Dahl RE, Puig-Antich J, Ryan ND et al. EEG sleep in adolescents with major depression: the role of suicidality and inpatient status. J Affect Disord 1990;19:63-75.

43. Kutcher S, Williamson P, Marton P, Szalai J. REM latency in endogenously depressed adolescents. $\mathrm{Br} \mathrm{J}$ Psychiatry 1992;161:399-402.
44. Zimmerman M, McGlinchey JB, Young D, Chelminski I. Diagnosing Major Depressive Disorder I. A psychometric evaluation of the DSM-IV symptom criteria. J Nerv Ment Dis 2006;194:158-163.

45. Benazzi F. The relationship of major depressive disorder to bipolar disorder: continuous or discontinuous? Curr Psychiatry Rep 2005;7:462-470.

46. Mitchell PB, Wilhelm K, Parker G, Austin MP, Rutgers P, MALHI GS. The clinical features of bipolar depression: a comparison with matched major depressive disorders. J Clin Psychiatry 2001;62:212-216.

47. Paykel ES, Rowan PR, Parker RR, Bhat AV. Response to phenelzine and amitriptyline in subtypes of outpatient depression. Arch Gen Psychiatry 1982;39:1041-1049.

48. Posternak MA. Biological markers of atypical depression. Harv Rev Psychiatry 2003;11:1-7.

49. Parker G, Malhi G, Hadzi-Pavlovic D, Parker K. Sleeping in? The impact of age and depressive sub-type on hypersomnia. J Affect Disord 2006;90:73-76.

50. Allen LS, Gorski RA. Sexual dimorphism of the anterior commissure and massa intermedia of the human brain. J Comp Neurol 1991;312:97-104.

51. Carskadon M, Dement WC. Normal Human Sleep: An Overview. In: M Kryger, T Roth, WC Dement, eds. Principle and Practice of Sleep Medicine Fourth Edition. Philadelphia: Elsevier Saunders, 2005:13-23.

52. Armitage R, Hudson A, Trivedi M, Rush AJ. Sex differences in the distribution of EEG frequencies during sleep: unipolar depressed outpatients. J Affect Disord 1995;34:121129.

53. Duncan WC, Pettigrew KD, Gillin JC. REM architecture changes in bipolar and unipolar patients. Am J Psychiatry 1979;136:1424-1427.

54. Foster FG, Kupfer DJ, Coble P, McPartland RJ. Rapid eye movement sleep density. An objective indicator in severe medical-depressive syndromes. Arch Gen Psychiatry 1976;33:1119-1123.

55. Gillin JC, Duncan W, Pettigrew KD, Frankel BL, Snyder F. Successful separation of depressed, normal, and insomniac subjects by EEG sleep data. Arch Gen Psychiatry 1979;36:85-90.

56. KUPFER DJ, Foster FG. Interval between onset of sleep and rapid-eye-movement sleep as an indicator of depression. Lancet 1972;2:684-686.

57. Coble P, Foster FG, Kupfer DJ. Electroencephalographic sleep diagnosis of primary depression. Arch Gen Psychiatry 1976;33:1124-1127.

58. Kupfer DJ, Foster FG, Reich L, Thompson KS, Weiss R. EEG sleep changes in REM sleep and clinical depression. Am J Psychiatry 1976;133:622-626.

59. BuYsSe DJ, KuPFER DJ. Diagnostic and research applications of electroencephlographic sleep studies in depression. Conceptual and methodological issues. J Nerv Ment Dis 1990; 178:405-414.

60. Rush AJ, Erman MK, Giles DE et al. Polysomnographic findings in recently drug-free and clinically remitted depressed patients. Arch Gen Psychiatry 1986;43:878884 .

61. Giles DE, Roffwarg HP, Schlesser MA, Rush AJ. Which endogenous depressive symptoms relate to REM latency reduction? Biol Psychiatry 1986;21:473-482.

62. Rush AJ, Erman MK, Schlesser MA et al. Alprazolam vs amitriptyline in depressions with reduced REM latencies. Arch Gen Psychiatry 1985;42:1154-1159.

63. Rush AJ, Giles DE, JarRett RJ et al. Reduced REM latency predicts response to tricyclic medication in depressed outpatients. Biol Psychiatry 1989;26:61-72. 
64. Heiligenstein JH, Faries DE, Rush AJ et al. Latency to rapid eye movement sleep as a predictor of treatment response to fluoxetine and placebo in nonpsychotic depressed outpatients. Psychiatry Res 1994;52:327-339.

65. Giles DE, Biggs MM, Roffwarg HP, Orsulak PJ, Rush AJ. Secondary depression: a comparison among subtypes. J Affect Disord 1987;12:251-258.

66. Rush AJ, Gullion CM, Basco MR, Jarrett RB, Trivedi MH. The Inventory of Depressive Symptomatology (IDS): psychometric properties. Psychol Med 1996;26:477-486.

67. Steiger A, von Bardeleben U, Wiedemann K, Holsboer F. Sleep EEG and nocturnal secretion of testosterone and cortisol in patients with major endogenous depression during acute phase and after remission. J Psychiatr Res 1991;25:169-177.

68. Giles DE, Biggs MM, Rush AJ, Roffwarg HP. Risk factors in families of unipolar depression. I. Psychiatric illness and reduced REM latency. J Affect Disord 1988;14:51-59.

69. Giles DE, Roffwarg HP, Kupfer DJ, Rush AJ, Biggs MA, Etzel BA. Secular trend in unipolar depression: a hypothesis. J Affect Disord 1989;16:71-75.

70. Rush AJ, Giles DE, Schlesser MA et al. Dexamethasone response, thyrotropin-releasing hormone stimulation, rapid eye movement latency, and subtypes of depression. Biol Psychiatry 1997;41:915-928.

71. Kupfer DJ, Frank E, Grochocinski VJ, Gregor M, McEACHRAN AB. Electroencephalographic sleep profiles in recurrent depression. A longitudinal investigation. Arch Gen Psychiatry 1988;45:678-681.

72. Kupfer DJ, Ehlers CL, Frank E, Grochocinski VJ, MCEACHRAN AB. EEG sleep profiles and recurrent depression. Biol Psychiatry 1991;30:641-655.

73. Giles DE, Jarrett RB, Roffwarg HP, Rush AJ. Reduced rapid eye movement latency: a predictor of recurrence in depression. Neuropsychopharmacology 1987;1:33-39.

74. Benca RM, Obermeyer WH, Thisted RA, Gillin JC. Sleep and psychiatric disorders. A meta-analysis. Arch Gen Psychiatry 1992;49:651-668.

75. Lauer C, Garcia D, Pollmacher T, Ozdaglar A, Krieg J. All-night EEG sleep in anxiety disorders and major depression. In: Horne J, ed. Sleep '90. Bochum: Pontanagel Press, 1991:29-31.

76. Armitage R, Hoffmann RF, Rush AJ. Biological rhythm disturbance in depression: temporal coherence of ultradian sleep EEG rhythms. Psychol Med 1999;29:14351448.

77. Armitage R, Calmoun JS, Rush AJ, Roffwarg HP. Comparison of the delta EEG in the first and second nonREM periods in depressed adults and normal controls. Psychiatry Res 1992;41:65-72.

78. Robert JJT, Hoffmann RF, Emslie $G$ et al. Sex and age differences in sleep macroarchitecture in childhood and adolescent depression. Sleep 2006;29:351-358.

79. Armitage R. Microarchitectural findings in sleep EEG in depression: diagnostic implications. Biol Psychiatry 1995;37:72-84.

80. Armitage R, Roffwarg HP, Rush AJ, Calhoun JS, Purdy DG, GILES DE. Digital period analysis of sleep EEG in depression. Biol Psychiatry 1992;31:52-68.

81. Liscombe MP, Hoffmann R, Trivedi M, Parker MK, Rush AJ, Armitage R. Quantitative EEG amplitude across REM sleep periods in depression: a preliminary study. J Psychiatry Neurosci 2002;27:40-46.

82. Armitage R, Roffwarg HP, Rush AJ. Digital period analysis of EEG in depression: periodicity, coherence, and interhemispheric relationships during sleep. Prog Neuropsychopharmacol Biol Psychiatry 1993;17:363-372.
83. Germain A, Nofzinger EA, Kupfer DJ, Buysse DJ. Neurobiology of non-REM sleep in depression: further evidence for hypofrontality and thalamic dysregulation. Am J Psychiatry 2004;161:1856-1863.

84. Perlis ML. Response to 'Do increases in beta EEG activity uniquely reflect insomnia?' (CH Bastien and $\mathrm{MH}$ Bonnet). Sleep Med Rev, 2001;5:381-385.

85. Pizzagalli DA, Nitschke JB, Oakes TR et al. Brain electrical tomography in depression: the importance of symptom severity, anxiety, and melancholic features. Soc Biol Psychiatry 2002;52:73-85.

86. Armitage R, Hoffmann R, Emslie G, Rintelmann J, Robert J. Sleep microarchitecture in childhood and adolescent depression: temporal coherence. Clin EEG Neurosci 2006;37:1-9.

87. Knott V, Mahoney C, Kennedy S, Evans K. EEG power, frequency, asymmetry and coherence in male depression. Psychiatry Res Neuroimaging 2001;106:123-140.

88. Fulton MK, Armitage R, Rush AJ. Sleep electroencephalographic coherence abnormalities in individuvals at high risk for depression: a pilot study. Biol Psychiatry 2000;47(7):618-625.

89. Morehouse RL, Kusumakar V, Kutcher SP, LeBlanc J, Armitage R. Temporal coherence in ultradian sleep EEG rhythms in a never-depressed, high-risk cohort of female adolescents. Biol Psychiatry 2002;51:446-456.

90. Modell S, Ising M, Holsboer F, Lauer CJ. The Munich Vulnerability Study on Affective Disorders: premorbid polysomnographic profile of affected high-risk probands. Biol Psychiatry 2005;58:694-699.

91. Cajochen C, Brunner DP, Krauchi K, Graw P, Wirz-Justice A. EEG and subjective sleepiness during extended wakefulness in seasonal affective disorder: circadian and homeostatic influences. Biol Psychiatry 2000;47:610-617.

92. Carrier J, Land S, Buysse DJ, Kupfer DJ, Monk TH. Age and gender effects on sleep EEG - power spectral density in the 'middle' years of life $(20 \mathrm{y}-60 \mathrm{y})$. Sleep 1999;22(suppl.):S223-S224.

93. Cole RJ, Kripke DF, Gruen W, Mullaney DJ, Gillin JC. Automatic sleep/wake identification from wrist activity. Sleep 1992;15:461-469.

94. Kupfer DJ, Frank E, McEachran AB, Grochocinski VJ. Delta sleep ratio: a biological correlate of early recurrence in unipolor affective disorder. Arch Gen Psychiatry 1990;47:1100-1105.

95. Antonimevic IA, Murck H, Frieboes RM, Uhr M, Steiger A. On the role of menopause for sleep-endocrine alterations associated with major depression. Psychoneuroendocrinology 2003;28:401-418.

96. Jindal RD, Friedman ES, Berman SR, Fasiczka AL, HowLAND RH, Thase ME. Effects of sertraline on sleep architecture in patients with depression. J Clin Psychopharmacol 2003;23:540-548.

97. Landolt HP, Gillin JC. Similar sleep EEG topography in middle-aged depressed patients and healthy controls. Sleep 2005;28:239-247.

98. Murck H, Uhr M, Ziegenbein M et al. Renin-AngiotensinAldosterone system, HPA-Axis and Sleep-EEG in unmedicated patients with depression after total sleep deprivation. Pharmacopsychiatry 2006;39:23-29.

99. Reynolds CF, Spiker DG, Hanin I, Kupfer DJ. Electroencephalographic sleep, aging and psychopathology: new data and state of the art. Biol Psychiatry 1983;18:139155.

100. Birmaher B, Heydl P. Biological studies in depressed children and adolescents. Int $\mathbf{J}$ Neuropsychopharmacol 2001;4:149-157. 


\section{Sleep and depression}

101. Armitage R, Hoffmann R, Emslie G, Rintelman J, Moore J, LEwIS K. Rest-activity cycles in childhood and adolescent depression. J Am Acad Child Adolesc Psychiatry 2004;43:761-769.

102. Reynolds CF, Kupfer DJ, Thase ME, et al. Sleep, gender, and depression: an analysis of gender effects on the electroencephalographic sleep of 302 depressed outpatients. Biol Psychiatry 1990;28:673-684.

103. Armitage R, Hoffmann R, Fitch T, Trivedi M, Rush AJ: Temporal characteristics of delta activity during NREM sleep in depressed outpatients and healthy adults: group and sex effects. Sleep, 2000;23(5):607-617.

104. Armitage R, Hoffmann R, Trivedi M, Rush AJ. Slow-wave activity in NREM sleep: sex and age effects in depressed outpatients and healthy controls. Psychiatry Res, 2000;95:201-213.

105. Armitage R, Hoffmann R, Robert JJ, Tekell JL. Gender differences in SWA response to a 3-hour sleep delay in healthy adults. Sleep 2005;28:A38.

106. Hollon SD, Shelton RC, Wisniewski S et al. Presenting characteristics of depressed outpatients as a function of recurrence: preliminary findings from the STAR*D clinical trial. J Psychiatr Res 2006;40:59-69.

107. Nierenberg AA, Trivedi MH, Fava M et al. Family history of mood disorder and characteristics of major depressive disorder: a STAR*D (sequenced treatment alternatives to relieve depression) study. J Psychiatr Res 2006 (in press).

108. Carter JD, Joyce PR, Mulder RT, Luty SE, McKenzie J. Gender differences in the presentation of depressed outpatients: a comparison of descriptive variables. J Affect Disord 2000;61:59-67.

109. Armitage R, Cole D, Suppes T, Ozcan M. Effects of clozapine on sleep in bipolar and schizoaffective illness. Prog Neuropsychopharmacol Biol Psychiatry 2004;28:10651070.

110. Mehl RC, O’Brien LM, Dreisbach JK, Mervis CB, Gozal D. Correlates of sleep and pediatric bipolar disorder. Sleep 2006;29:193-197.

111. Bauer M, Grof P, Rasgon N, Bschor T, Glenn T, Whybrow PC. Temporal relation between sleep and mood in patients with bipolar disorder. Bipolar Disord 2006;8: $160-167$

112. Perlman CA, Johnson SL, Mellman TA. The prospective impact of sleep duration on depression and mania. Bipolar Disord 2006;8:271-274.

113. Kawa I, Carter JD, Joyce PR et al. Gender differences in bipolar disorder: age of onset, course, comorbidity, and symptom presentation. Bipolar Disord 2005;7:119 125 . 